\title{
Current \& Future Gamma Ray Astronomy Related to INTEGRAL
}

\section{N. Gehrels*}

Astroparticle Physics Division, NASA/Goddard Space Flight Center, Greenbelt, MD 20771, USA

E-mail: neil.gehrels@nasa.gov

\section{J. K. Cannizzo}

CRESST/Joint Center for Astrophysics, Univ. of Maryland, Baltimore County, Baltimore, MD

21250, USA

E-mail: john.k.cannizzo@nasa.gov

The INTEGRAL mission and other high energy missions have provided key insights into a wide variety of topics in astronomy, ranging from local to galactic to extragalactic. High-energy instruments on INTEGRAL have been greatly supported by gamma-ray missions such as Swift/BAT, Fermi/LAT, and RXTE/HEXTE. In this review we highlight four recent discoveries in high energy astrophysics: the clear detection of cyclotron lines in absorption in galactic transients, fluctuations in the power output of the Crab nebula - previously thought to be a standard candle, the jetted tidal disruption event Sw1644, and the $\log N-\log S$ distribution of Compton thick AGN. For each topic we first provide a general physical background for context, and then discuss the missions that are supporting INTEGRAL on these topics. The unexpected nature of some of these discoveries continues to challenge our understanding of astrophysical processes, and underscores the necessity of future, more detailed observations.

"An INTEGRAL view of the high-energy sky (the first 10 years)" 9th INTEGRAL Workshop and celebration of the 10th anniversary of the launch,

October 15 - 19, 2012

Bibliotheque Nationale de France, Paris, France

\footnotetext{
* Speaker.
} 


\section{Selected Science Highlights}

\subsection{Cyclotron lines}
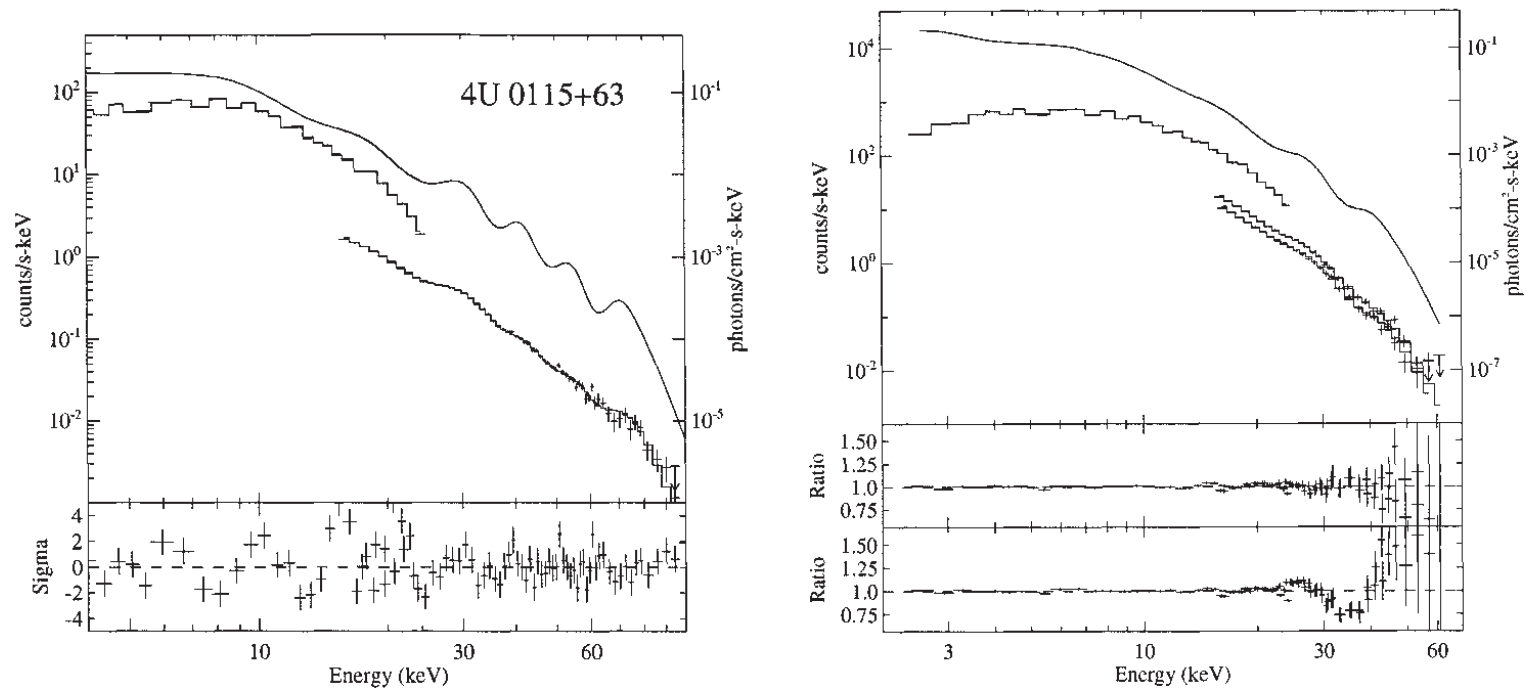

Figure 1: $R X T E / P C A$ and HEXTE detection of cyclotron absorption lines in 4U0115+63 (Heindl et al. 2000), a transient accreting X-ray pulsar $(P=3.6 \mathrm{~s})$ in an eccentric $24.3 \mathrm{~d}$ orbit.

Cyclotron lines can appear in the spectra of accreting neutron stars (NSs) in binary systems if the NS harbors a magnetic field $B \sim 10^{11}-10^{13} \mathrm{G}$ (Harding \& Lai 2006). X-ray pulsars accrete either via a stellar wind or Roche lobe overflow through an accretion disk. The NS magnetic field channels the flow onto the magnetic poles with terminal free-fall velocities $(G M / R c) \sim 0.4 c$. The allowed transition levels between states in cyclotron absorption is given by $\varepsilon_{n} /\left(m_{e} c^{2}\right)=[(1+$ $\left.\left.2 n\left(B / B_{\mathrm{QED}}\right) \sin ^{2} \theta\right)^{1 / 2}-1\right] / \sin ^{2} \theta$, where $n$ is the excitation state, $\theta$ is the angle between the photon propagation angle and magnetic field, and $B_{\mathrm{QED}}=m_{e}^{2} c^{2} /(e \hbar)=4.414 \times 10^{13} \mathrm{G}$. The critical field $B_{\mathrm{QED}}$ represents the point at which the nonrelativistic Landau energy $\hbar e B /\left(m_{e} c\right)$ equals the electron rest energy $m_{e} c^{2}$. For $B / B_{\mathrm{QED}} \ll 1$ this formula reduces to the "12-B-12 rule", $\varepsilon_{n}=11.57 n B_{12} \mathrm{keV}$, where $B_{12}=B / 10^{12} \mathrm{G}$. In practice, the strong gravitational redshift from the NS surface introduces an additional factor $(1+z)^{-1}$, where typically $z \simeq 0.3$ for NSs. The source boasting the greatest number of lines is $4 \mathrm{U} 0115+63$ with five features detected (Heindl et al. 2000). The two panels in Figure 1 show RXTE data for $4 \mathrm{U} 0115+63$, best fit spectra for phase A and B (left and right, respectively), where $\mathrm{A}$ is phase bin $0.32-0.38$ (the falling edge of the main pulse), and B is phase bin $0.70-0.76$ (the falling edge of the secondary pulse). Five distinct absorption features are seen in the phase A data, whereas three are seen in the phase B data. INTEGRAL has also provided a valuable contribution to the field with the JEM-X/ISGRI detection of cyclotron lines from the highmass X-ray transient V0332+53 during decline from outburst (Mowlavi et al. 2006). Tsygankov et al. (2006) present a study of V0332+53 which shows more clearly the displacement of cyclotron line energies, possibly due to a change of the accretion column. 


\subsection{Inconstancy of the Crab Nebula}

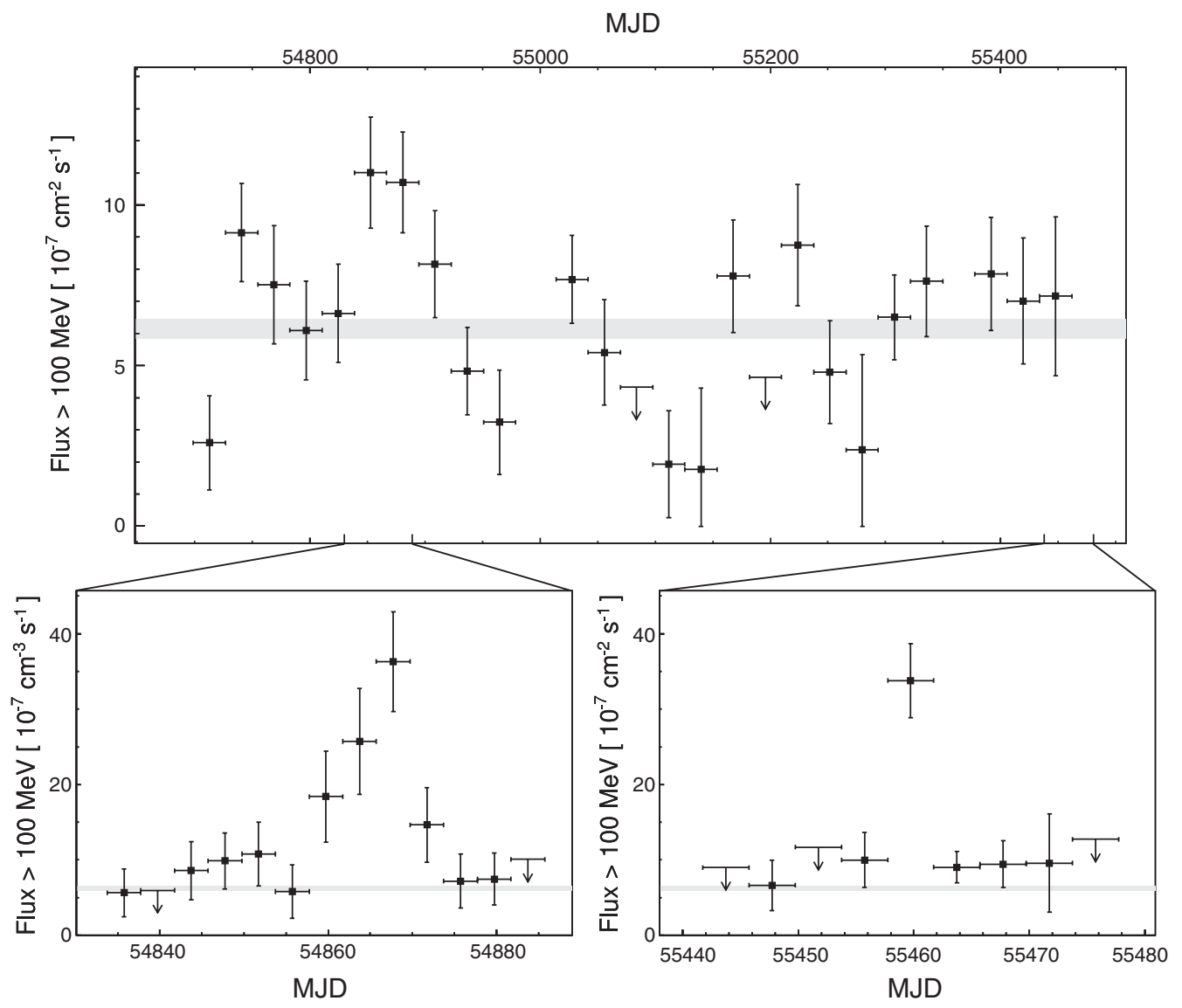

Figure 2: Gamma-ray flux $>100 \mathrm{MeV}$ of the synchrotron component of the Crab Nebula (Abdo et al. 2011). Top panel shows the flux in $4 \mathrm{wk}$ bins. Gray band indicates the average flux. Bottom panel shows the flux in $4 \mathrm{~d}$ bins during selected intervals.

The Crab nebula is the result of a supernova recorded in $1054 \mathrm{AD}$. At energies above $\sim 30 \mathrm{keV}$ it is the brightest persistent source in the sky. The nebula is powered by a pulsar with spin period $P=0.033 \mathrm{~s}$ and period derivative $\dot{P}=4.2 \times 10^{-13} \mathrm{~s} \mathrm{~s}^{-1}$. The observed power output $\dot{E}_{\text {out }}$ for the nebula, kinetic plus radiation, is $\sim 5 \times 10^{38} \mathrm{erg} \mathrm{s}^{-1}$. The expanding ejecta which make up the nebula are thought to be powered primarily by the spin-down energy from the pulsar $\dot{E}_{\text {loss }}=I \Omega \dot{\Omega}$, where $I$ is the neutron star moment of inertia and $\Omega$ the rotational rate $2 \pi / P$. Evaluating the loss rate assuming neutron star parameters $M=1.4 M_{\odot}, R=12 \mathrm{~km}, I=1.4 \times 10^{45} \mathrm{~g} \mathrm{~cm}^{2}$, and the observed spin-down rate yields $\dot{E}_{\text {loss }}=6.4 \times 10^{38} \mathrm{erg} \mathrm{s}^{-1}$, in line with the total nebula power output. Based on these simple considerations, the recent Fermi/LAT finding of large GeV flares coming from the Crab nebula was totally unexpected (Figure 2, from Abdo et al. 2011; see also Tavani et al. 2011). Unexpected variability is also seen in lower energy gamma-ray measurements in Figure 3 which shows composite long term light curves of the Crab nebula in various high energy bands (from Wilson-Hodge et al. 2011). The Crab was previously thought to be a steady source and was used as a calibration standard. Now we learn that GeV flares on week-long timescales occur and that 
$\sim 20 \%$ variability on year-long timescales is detected. Observations from AGILE, Fermi, Swift, RXTE and INTEGRAL have been combined to make these discoveries.

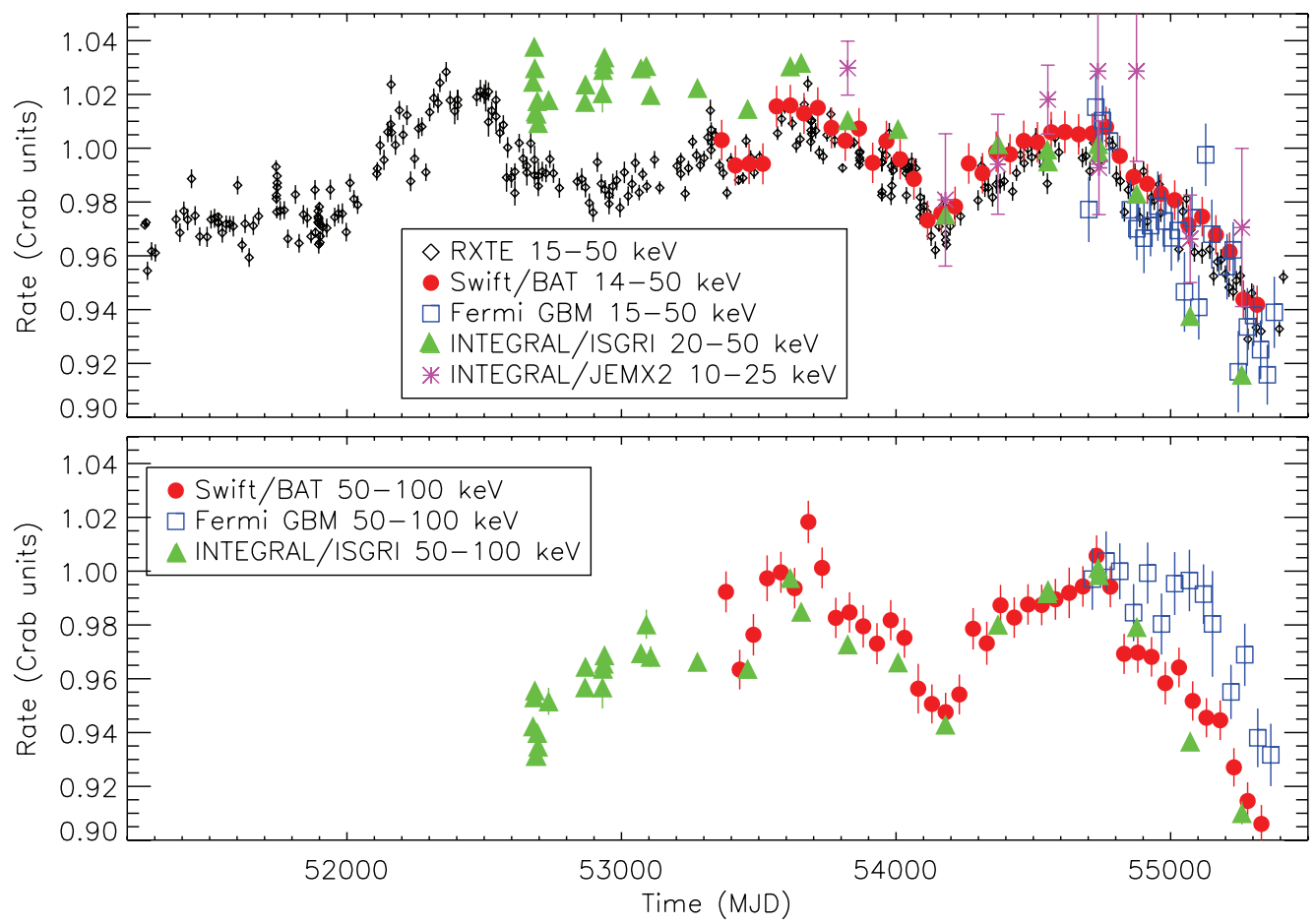

Figure 3: Composite light curves of the Crab nebula from RXTE/PCA ( $15-50 \mathrm{keV}$ : black diamonds), Swift/BAT (top: $14-50 \mathrm{keV}$, bottom: $50-100 \mathrm{keV}$ : red filled circles), Fermi/GBM (top: $15-50 \mathrm{keV}$, bottom: $50-100 \mathrm{keV}$ : open blue squares), INTEGRAL/ISGRI (top: $20-50 \mathrm{keV}$, Bottom: $50-100 \mathrm{keV}$ : green triangles), and INTEGRAL/JEM-X2 (10-25 keV) (Wilson-Hodge et al. 2011). Each data set has been normalized to its mean rate during MJD $54690-54790$.

\subsection{A Jetted Tidal Disruption Event}

Tidal disruption events (TDEs) are caused by the tidal disruption of stars that venture too close to the massive black holes (MBHs) at the centers of galaxies (Rees 1988, Phinney 1989). Prior to March 2011, nearly all our observational information was based on optical/UV studies (Gezari et al. 2006, 2008) or long-term X-ray data with poor time sampling (Komossa et al. 2004). This changed with the discovery by Swift of GRB 110328A/Swift J1644 (= Sw1644), a TDE viewed down the jet axis of a MBH in the nucleus of a galaxy at redshift $z=0.35$ (Bloom et al. 2011, Burrows et al. 2011, Levan et al. 2011, Zauderer et al. 2011). In hindsight, it is surprising that there was almost no prior appreciation (e.g., Giannios \& Metzger 2011) that one might have a TDE viewed down the axis of the black hole jet, such that boosting would occur. For Sw1644 only the boosted, jetted emission was seen; despite early optimism that the thermal flux from the accretion disk might be observable in XMM-Newton observations at the $\sim 1 \%$ level, it was not detected with any statistical significance. Continued observations for over $1 \mathrm{yr}$ with the Swift/XRT have shown an apparent long term decay law $L_{x} \propto t^{-\alpha}$ with $\alpha \simeq-1.3$, (Figure 4), which may be consistent with the decay 

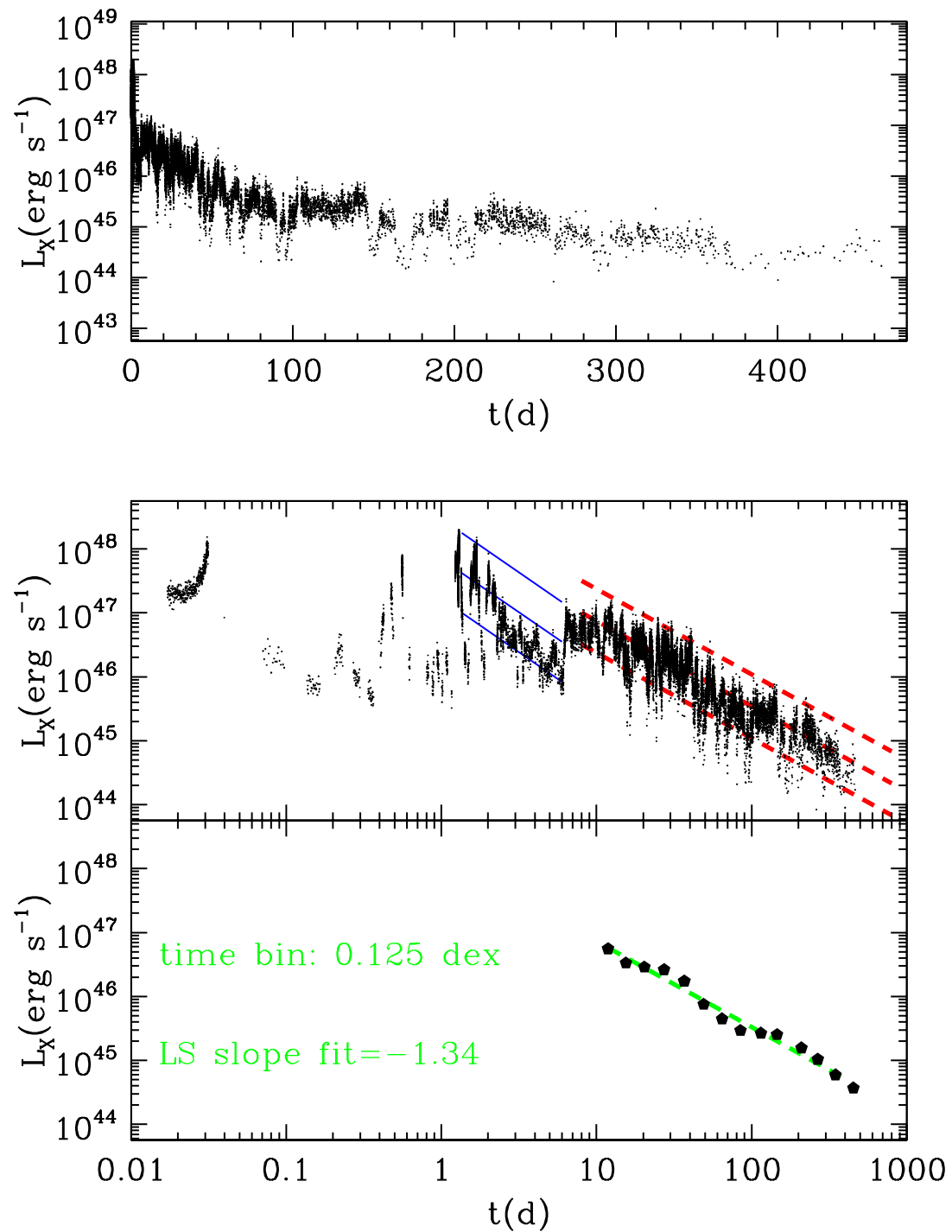

Figure 4: The long term Swift/XRT X-ray light curve of Sw1644 through 1 July 2012 from the public Swift/XRT website (as described in Evans et al. 2007, 2009). The top panel shows the data as $\log L_{X}-t$, whereas the middle and bottom show $\log L_{X}-\log t$. A least squares fit to the decay slope with $\Delta \log t=0.125$ binning in time gives -1.34 for the decay slope. Other binnings give similar rates.

of a freely expanding, advectively dominated slim disk (Cannizzo, Troja, \& Lodato 2011). This decay law appears to hold as early as $t \simeq 10 \mathrm{~d}$, indicating that the dividing point between "stellar fallback" $\left(L \propto t^{-5 / 3}\right)$ and "disk accretion" $\left(L \propto t^{-4 / 3}\right)$ (Phinney 1989, Cannizzo, Lee, \& Goodman 1990) may have been at $\lesssim 10 \mathrm{~d}$, indicative of a deeply plunging disruption. This is in contrast to the more probable event where a disruption occurs close to the classical tidal disruption radius, in which case the dividing point would lie at years to decades. If Sw1644 was deeply plunging, that may also be part of the reason it was a powerful, jetted TDE. 


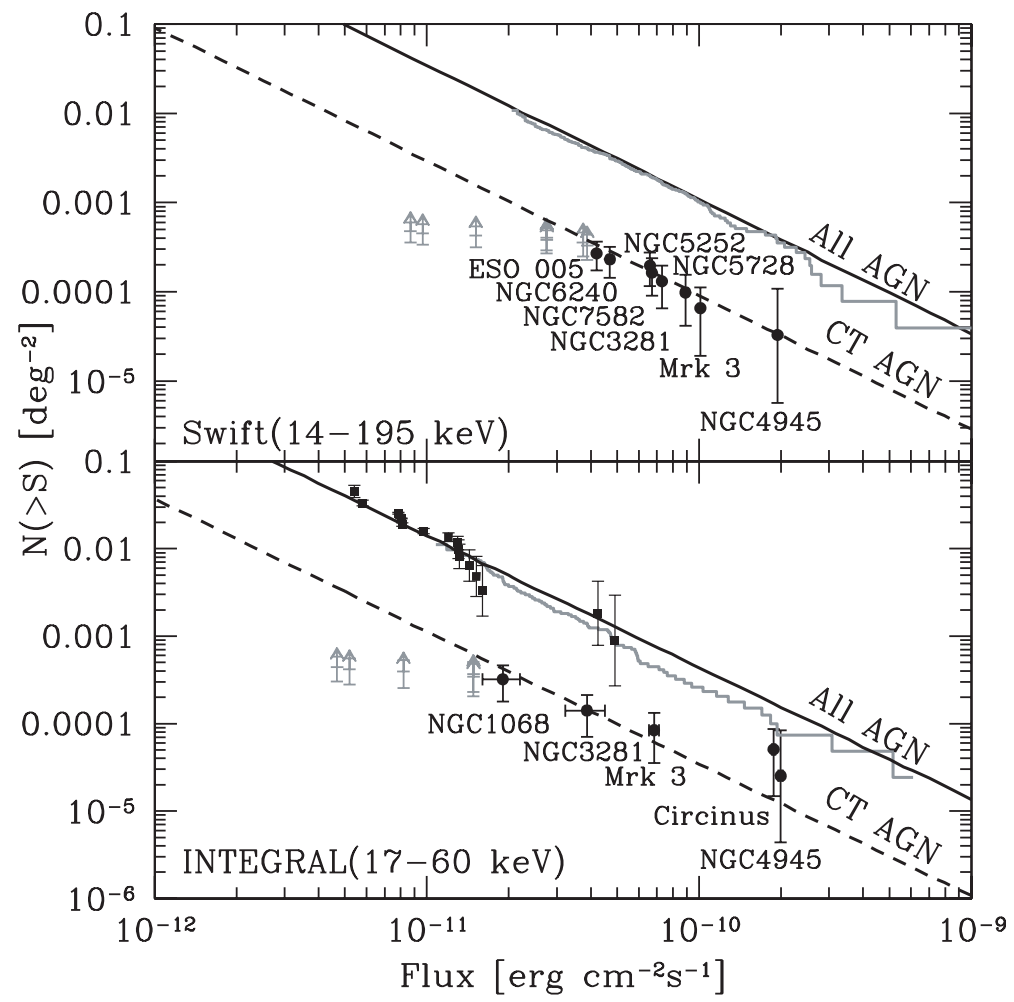

Figure 5: The $\log N-\log S$ distribution for $A G N$ detected at high energies (Treister et al. 2009). CT AGN detected with Swift (top panel) and INTEGRAL (bottom panel). Black solid lines show the expected AGN $\log N-\log S$ from the population synthesis model of Treister \& Urry (2005). Dashed lines mark the Euclidean slope normalized to the number of Swift and INTEGRAL CT AGNs. Gray lower limits indicate previously known transmission-dominated AGN with hard X-ray observations, not detected in the Swift or INTEGRAL surveys.

\subsection{A Census of Compton Thick AGN}

Until recently heavy absorption toward the centers of galaxies has limited our view of accretion onto supermassive black holes. Locally the ratio of obscured to unobscured sources is about four to one. The most heavily obscured AGNs are those for which the HI column density exceeds the inverse Thomson cross section $\sim 1.5 \times 10^{24} \mathrm{~cm}^{-2}$. These are termed "Compton thick" (CT) AGNs. Now, thanks to wide-area surveys at high energies it has become possible to study a well defined sample of CT AGNs and to address such issues as the fraction of AGNs contributing to the cosmic X-ray background (XRB). Figure 5 shows a $\log N-\log S$ distribution for AGN detected at high energies derived from Swift/BAT and INTEGRAL/IBIS surveys (Treister et al. 2009). Treister et al. determine the contribution of CT AGNs to the XRB to be small, about $9 \%$.

NUSTAR will write the next chapter in this line of research with its $\sim 100$ times better sensitivity; for a small patch on the sky it will burrow down to a flux level $\sim 100$ times smaller than indicated in Figure 5, and delineate any changes in the $\log N-\log S$ relationship at flux levels $\lesssim 10^{-12} \mathrm{erg} \mathrm{cm}^{-2} \mathrm{~s}^{-1}$. 


\section{Forthcoming hard X-ray/soft gamma-ray missions}

Four other missions are noteworthy for the potentially high impact science they could produce:

ASTROSAT: An Indian mission, scheduled for launch in 2013. It has five instruments: twin 40 cm UV imaging telescopes, three large area Xe proportional counters $(3-80 \mathrm{keV})$ with effective area $\sim 6000 \mathrm{~cm}^{2}$ at $10 \mathrm{keV}$, a soft X-ray telescope $(0.3-8 \mathrm{keV})$ with area $\sim 200 \mathrm{~cm}^{2}$ at $1 \mathrm{keV}$, a CZT imager $(10-150 \mathrm{keV})$ with area $\sim 1000 \mathrm{~cm}^{2}$, and a sky monitor capable of scanning the sky once every $6 \mathrm{hr}$. It will monitor the X-ray sky for new transients, provide surveys in the hard X-ray and UV, carry out broad band spectroscopic studies, as well as detailed timing studies of periodic and nonperiodic sources.

Hard X-ray Modulation Telescope $=$ HMXT: A Chinese mission, scheduled for launch between 2014 and 2016. Its main instrument is an array of 18 phoswich detectors collimated to $6^{\circ} \times 1^{\circ}$ overlapping fields of view (FoV). The primary NaI detectors each have an effective area $286 \mathrm{~cm}^{2}$ and will cover $20-200 \mathrm{keV}$. The total effective combination will have a $6^{\circ} \times 6^{\circ} \mathrm{FoV}$ and effective area $\sim 5000 \mathrm{~cm}^{2}$. It will have excellent angular and energy resolution.

Astro-H: A Japanese mission, scheduled for launch in 2014. It will carry hard and soft X-ray focusing telescopes, as well as a collimated $\gamma$-ray $\mathrm{Si} / \mathrm{CdTe}$ instrument with sensitivity $4-600$ $\mathrm{keV}$. Astro- $H$ will address such questions as "How do structures such as galaxies and clusters of galaxies form and evolve?" and "How do supermassive black holes feed back energy into galaxies and clusters?" and "What is the nature of dark matter and dark energy?".

ACT Compton Telescope: A future mission concept under study. This will have a larger FoV than most other missions, and a large collecting area, utilizing Si, Ge, and CZT stacks. It will have a medium energy range of $0.2-10 \mathrm{MeV}$ and be able to study nuclear $\gamma$-ray lines in pulsars and blazars. Its primary mission will be to uncover how supernovae and other stellar explosions work to create the elements.

\section{Summary}

The last decade has overseen a wealth of unexpected results in high energy astrophysics, as typified by discoveries from INTEGRAL and other high energy observatories. The universe has been revealed to be a more wondrous and sometimes violent place than previously imagined. Some of the results discussed were unexpected, such as the variability of the Crab nebula, whereas other results have complemented those from lower energy wavebands. Given our incomplete understanding of some of these phenomena, there is enormous opportunity for more detailed follow-up. The future is bright for high energy astrophysics.

\section{References}

[1] A. A. Abdo, et al., Gamma-Ray Flares from the Crab Nebula. Science 331739 [2011].

[2] J. S. Bloom, et al., A Possible Relativistic Jetted Outburst from a Massive Black Hole Fed by a Tidally Disrupted Star. Science 333203 [2011].

[3] D. N. Burrows, et al., Relativistic jet activity from the tidal disruption of a star by a massive black hole. Nature 476421 [2011]. 
[4] J. K. Cannizzo, H. M. Lee, \& J. Goodman, The Disk Accretion of a Tidally Disrupted Star onto a Massive Black Hole. ApJ 35138 [1990].

[5] J. K. Cannizzo, E. Troja, \& G. Lodato, GRB 110328A/Swift J164449.3 + 573451: The Tidal Obliteration of a Deeply Plunging Star? ApJ 74232 [2011].

[6] P. A. Evans, et al., An online repository of Swift/XRT light curves of $\gamma$-ray bursts. $A \& A 469379$ [2007].

[7] P. A. Evans, et al., Methods and results of an automatic analysis of a complete sample of Swift-XRT observations of GRBs. MNRAS 3971177 [2009].

[8] S. Gezari, et al., Ultraviolet Detection of the Tidal Disruption of a Star by a Massive Black Hole. ApJ 653 L25 [2006].

[9] S. Gezari, et al., UV/Optical Detections of Candidate Tidal Disruption Events by GALEX and CFHTLS1. ApJ 676944 [2008].

[10] D. Giannios, \& B. D. Metzger, Radio transients from stellar tidal disruption by massive black holes. MNRAS 4162102 [2011].

[11] A. K. Harding, \& D. Lai, Physics of strongly magnetized neutron stars. Rep Prog Phys 692631 [2006].

[12] W. A. Heindl, W. Coburn, D. E. Gruber, M. Pelling, R. E. Rothschild, J. Wilms, K. Pottschmidt, \& R. Staubert, Multiple Cyclotron Lines in the Spectrum of 4U 0115+63. in Proc. 5th Compton Symp., ed. M. L. McConnell, \& J. M. Ryan (New York: AIP), AIP Conf. Proc. 5101230 [2000].

[13] S. Komossa, J. Halpern, N. Schartel, G. Hasinger, M. Santos-Lleo, \& P. Predehl, A Huge Drop in the X-Ray Luminosity of the Nonactive Galaxy RX J1242.6 - 1119A, and the First Postflare Spectrum: Testing the Tidal Disruption Scenario. ApJ 603 L17 [2004].

[14] A. J. Levan, et al., An Extremely Luminous Panchromatic Outburst from the Nucleus of a Distant Galaxy. Science 333199 [2011].

[15] N. Mowlavi, et al., INTEGRAL observation of the high-mass X-ray transient V0332 + 53 during the 2005 outburst decline. A\&A 451187 [2006].

[16] E. S. Phinney, Manifestations of a Massive Black Hole in the Galactic Center. IAU Symposium - The Center of the Galaxy ed. M. Morris, 136543 [1989].

[17] M. J. Rees, Tidal disruption of stars by black holes of $10^{6}-10^{8}$ solar masses in nearby galaxies. Nature 333523 [1988].

[18] M. Tavani, et al., Discovery of Powerful Gamma-Ray Flares from the Crab Nebula. Science 331736 [2011].

[19] E. Treister, C. M. Urry, Active Galactic Nuclei Unification and the X-Ray Background. ApJ 630115 [2005].

[20] E. Treister, C. M. Urry, \& S. Virani, The Space Density of Compton-Thick Active Galactic Nucleus and the X-Ray Background. ApJ 696110 [2009].

[21] S. S. Tsygankov, A. A. Lutovinov, E. M. Churazov, \& R. A. Sunyaev, V0332+53 in the outburst of 2004-2005: luminosity dependence of the cyclotron line and pulse profile. MNRAS 37119 [2006].

[22] C. A. Wilson-Hodge, et al., When a standard candle flickers. ApJ 727 L40 [2011].

[23] B. A. Zauderer, et al., Birth of a relativistic outflow in the unusual $\gamma$-ray transient Swift J164449.3 + 573451. Nature 476425 [2011]. 